\title{
Monte Carlo Simulations for Applications in Electron Backscatter Diffraction.
}

\author{
Aimo Winkelmann ${ }^{1}$, Francesc Salvat-Pujol ${ }^{2}$ and Wolfgang S. M. Werner ${ }^{2}$ \\ 1. Max-Planck-Institute of Mikrostructure Physics, Weinberg 2, D-06120 Halle, Germany \\ 2. Vienna University of Technology, Wiedner Hauptstraße 8-10, A-1040 Vienna, Austria
}

Electron Backscatter Diffraction (EBSD) in the scanning electron microscope (SEM) delivers important crystallographic information, which is contained in the Kikuchi patterns of backscattered electrons (BSE) near the incident energy $E_{0}$. To describe details of of EBSD pattern generation, Monte Carlo (MC) simulations of the BSE energy spectrum can provide useful additional information. However, the need to correctly describe the low loss energy range near $E_{0}$ limits the applicability of the "continuous slowing down approximation" (CSDA) [1], often used to describe inelastic scattering in SEM MC simulations [2]. In contrast, simulations including discrete inelastic scattering processes based on the differential inverse inelastic mean free path (DIIMFP) have been successfully applied for the quantitative description of reflection energy loss spectroscopy (REELS) [1].

In order to compare both approaches, we carried out $\mathrm{MC}$ simulations for backscattering of $E_{0}=10 \mathrm{keV}$ electrons incident at $70^{\circ}$ on a Si sample. In Fig. 1, we show the BSE spectra obtained using the CSDA in Casino 2.48 [2], and with discrete inelastic scattering using the REELS code from [7]. While both simulations result in a total integrated backscattering coefficient of $\eta=0.6$, the energy distributions near $\mathrm{E}_{0}$ are drastically different. In contrast to the CSDA simulation, only the REELS simulation correctly reproduces the elastic backscattering peak at the primary beam energy, as well as the discrete plasmon loss features observed in energy resolved Kikuchi band measurements of $\mathrm{Si}$ [3].

In order to extract diffraction-relevant information from the MC results, we classify the electron trajectories into two groups. The first group ("localized BSE") has a "large-angle" scattering as the last elastic event, followed by at most $n_{\text {ie }}$ inelastic events. These electrons are assumed to be scattered near an atomic nucleus, giving the crystal structure information in the EBSD pattern while all other types of trajectories are taken as originating on average from anywhere in a unit cell ("uniform BSE") and thus are assigned to a featureless background [4]. Here we take the limit of $n_{\mathrm{ie}}=0$ (no inelastic scattering after the large angle event) which assumes that each inelastic event leads to total randomization of the scattering location within the crystal unit cell. The "large-angle" electron-atom scattering is defined as resulting in a transfer of recoil energy exceeding a critical value $E_{\mathrm{R}}$, which is treated as a free parameter in this investigation. The resulting depth distributions for $E_{\mathrm{R}}=0.05 \mathrm{eV}$ are shown in Fig. 2. In this case, the distribution $N(z)$ of the "localized BSE" (yellow) would contribute $8 \%$ to the total intensity and can be fitted by $N(z)=N_{0} \exp (z / \lambda)$ with a mean depth of $\lambda=12 \mathrm{~nm}$. For comparison, the gray histogram shows the maximum penetration depth $z$ reached by the trajectories assigned to background electrons. In order to see the influence of the specific value of $E_{R}$, Table 1 summarizes results for $0.001 \mathrm{eV}<E_{\mathrm{R}}<0.2 \mathrm{eV}$. In all cases, the depth distribution of the localized BSE is well described by an exponential decay. For $E_{\mathrm{R}}=0.001 \mathrm{eV}$ and $E_{\mathrm{R}}=0.2 \mathrm{eV}$, the localized fraction (determined by $N_{0}$ ) approaches the limits of a few percent compatible with signal-to-background ratios seen in experimental EBSD patterns. All in all, this enables us to estimate a depth sensitivity $(\lambda)$ of EBSD from $\mathrm{Si}$ which is near 10 to $12 \mathrm{~nm}$ for $10 \mathrm{keV}$ electrons in a typical geometry with 70 degree incidence angle. For $20 \mathrm{kV}$ electrons, we obtain a decay constant of about $22 \mathrm{~nm}$. 
Our results can be interpreted by relatively simple models: Momentum transfer to atoms bound in a crystal is related to the physics of the Mössbauer effect [5], from which one expects that $E_{\mathrm{R}}$ should be of the order of representative phonon energies in the material (typically $<100 \mathrm{meV}$ ). The depth sensitivity in our model should be governed by the total inelastic mean free path, which is about $17 \mathrm{~nm}$ for $10 \mathrm{keV}$ in $\mathrm{Si}$ [6]. The effective values of $\lambda=10 . .12 \mathrm{~nm}$ (measured along $z$ ) reflect the inclination of the exit paths of the BSE in experimental EBSD measurements. In future investigations, the incoherent source distributions obtained from a MC simulation will be included in dynamical electron diffraction calculations, which should provide an improved description of EBSD patterns, including the information which is present in the raw backscattered signal.

References:

[1] W S M Werner, Surf. Interf. Anal. 31 (2001) 141

[2] D Drouin et al, Scanning 92 (2007) 92

[3] A Winkelmann, K Aizel, M Vos, New Journal of Physics 12 (2010) 053001

[4] A Winkelmann, M Vos, Ultramicroscopy 125 (2013) 66

[5] H J Lipkin, Ann. Phys. 9 (1960) 332

[6] S Tanuma, C J Powell, D R Penn, Surf. Interf. Anal. 43 (2011) 689

[7] F Salvat-Pujol, W S M Werner, Surf. Interf. Anal., in press, DOI: 10.1002/sia.5175

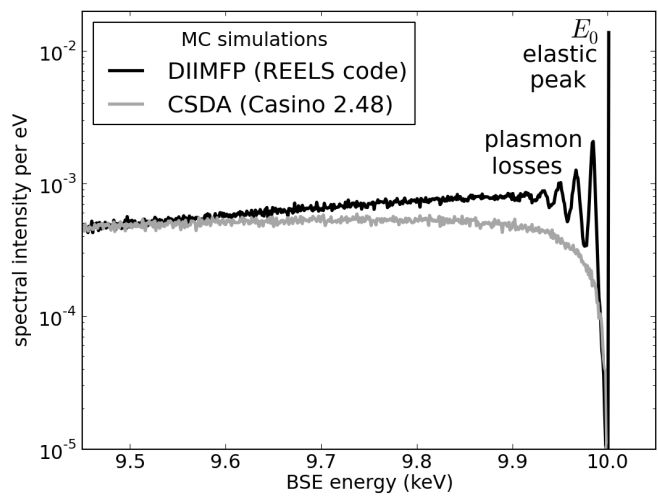

Figure 1. Simulated BSE spectra for Si, $E_{0}=10 \mathrm{keV}, 70^{\circ}$ incidence. Integrated spectral intensity normalized to 1.0 for $50 \mathrm{eV}-10 \mathrm{keV}$. For details see text.

Table 1. Effective incoherent source depth distribution and fraction of localized backscattering (relative to total BSE) for different values of the critical recoil energy $E_{R}$. The source depth distributions are fitted to $N(z)=N_{0} \exp (z / \lambda)$

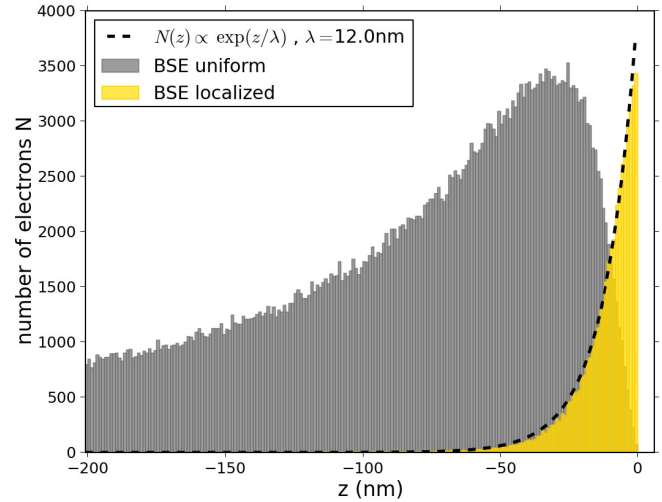

Figure 2. Yellow: Depth distribution of the "localized BSE" forming the Kikuchi pattern (dashed line: fit). Gray: Maximum z reached by "uniform BSE" in the featureless background.

\begin{tabular}{|c|l|c|}
\hline $\begin{array}{c}\text { critical recoil } \\
\text { energy } \mathbf{E}_{\mathbf{R}} \mathbf{( e V )}\end{array}$ & $\begin{array}{c}\text { Mean source } \\
\text { depth } \boldsymbol{\lambda}(\mathbf{n m})\end{array}$ & $\begin{array}{c}\text { Fraction of } \\
\text { localized BSE }\end{array}$ \\
\hline 0.200 & 13.2 & $2 \%$ \\
\hline 0.100 & 12.4 & $5 \%$ \\
\hline 0.050 & 12.0 & $8 \%$ \\
\hline 0.010 & 9.6 & $19 \%$ \\
\hline 0.001 & 6.4 & $44 \%$ \\
\hline
\end{tabular}

